\title{
Functionalized nanostructures for enhanced photocatalytic performance under solar light
}

\author{
Liejin Guo*, Dengwei Jing*, Maochang Liu, Yubin Chen, Shaohua Shen, Jinwen Shi \\ and Kai Zhang
}

\author{
Review \\ Address: \\ International Research Center for Renewable Energy \& State Key \\ Laboratory of Multiphase Flow in Power Engineering, Xi'an Jiaotong \\ University Xi'an 710049, China \\ Email: \\ Liejin Guo* - lj-guo@mail.xjtu.edu.cn; \\ Dengwei Jing ${ }^{*}$ - dwjing@mail.xjtu.edu.cn \\ * Corresponding author \\ Keywords: \\ functionalized nanostructures; hydrogen; photocatalysis; \\ photocatalytic; solar light
}

Beilstein J. Nanotechnol. 2014, 5, 994-1004.

doi:10.3762/bjnano.5.113

Received: 20 February 2014

Accepted: 02 June 2014

Published: 09 July 2014

This article is part of the Thematic Series "Photocatalysis".

Guest Editor: R. Xu

(c) 2014 Guo et al; licensee Beilstein-Institut.

License and terms: see end of document.

\begin{abstract}
Photocatalytic hydrogen production from water has been considered to be one of the most promising solar-to-hydrogen conversion technologies. In the last decade, various functionalized nanostructures were designed to address the primary requirements for an efficient photocatalytic generation of hydrogen by using solar energy: visible-light activity, chemical stability, appropriate bandedge characteristics, and potential for low-cost fabrication. Our aim is to present a short review of our recent attempts that center on the above requirements. We begin with a brief introduction of photocatalysts coupling two or more semiconductors, followed by a further discussion of the heterostructures with improved matching of both band structures and crystal lattices. We then elaborate on the heterostructure design of the targeted materials from macroscopic regulation of compositions and phases, to the more precise control at the nanoscale, i.e., materials with the same compositions but different phases with certain band alignment. We conclude this review with perspectives on nanostructure design that might direct future research of this technology.
\end{abstract}

\section{Review \\ Introduction}

The increasing energy demand as well as the serious environmental contamination caused by the usage of fossil fuels give rise to the necessity to develop clean alternative fuels. Hydrogen, as a pollution-free and storable energy fuel, is a promising substitute of fossil fuels. Nowadays hydrogen is mainly manu- factured from hydrocarbons such as fossil fuels, which limits its wide utilization. Therefore, the ability to economically and efficiently harvest hydrogen from renewable energies is central to advances in many areas and should be the fundamental research issue [1-4]. Photocatalytic hydrogen production from water by 
using solar energy is one of the most acceptable routes for this aim, since only abundant water and solar energy are needed for hydrogen production in the process. If the economic viability for industrial application is successfully satisfied, it will ultimately solve the energy and environmental problems $[5,6]$.

Since the first report by Fujishima and Honda in 1972 [7], hydrogen production from water over semiconducting powders or films by using solar energy has been extensively studied. Thermodynamically, the reaction of producing hydrogen and oxygen from water splitting has a standard Gibbs free energy $(\Delta G)$ of $237 \mathrm{~kJ} / \mathrm{mol}$ and is therefore an uphill reaction. Energy input is therefore indispensible for this reaction to proceed. In principle, the photocatalytic reaction over semiconductors is triggered by the direct absorption of a photon by the band gap of semiconductor materials (Figure 1). Upon photon excitation, the photogenerated charges move to the surface of semiconductor particles where photocatalytic reactions occur. Consequently, the efficiency of photocatalytic water splitting is closely affected by the band structure of the semiconductors [8].

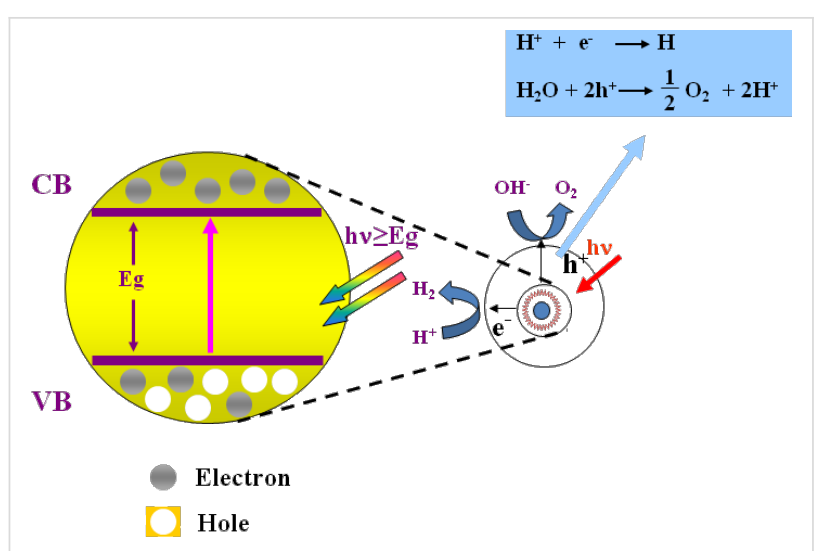

Figure 1: Basic principle for overall water splitting over semiconductor photocatalysts. Reprinted with permission from [6]. Copyright (2010) Elsevier.

The band gap of semiconductor photocatalysts must be larger than the potential of water electrolysis to meet the energetic requirement for overall water splitting $(1.23 \mathrm{eV}$, corresponding to an absorption threshold of $1000 \mathrm{~nm}$ ). In particular, the bottom level of the conduction band (CB) must be more negative than the reduction potential of water, while the top level of the valance band (VB) should be more positive than the oxidation potential of water. In order to utilize the abundant visible light from the sun, the band gap of photocatalysts has to be less than $3.0 \mathrm{eV}$ (corresponding to an absorption threshold larger than $420 \mathrm{~nm}$ ). Efficient utilization of these huge amounts of "low energy" photons is crucial to the realization of commercial solar photocatalytic hydrogen production. To this end, band engineering is necessary to design semiconductor photocata- lysts with satisfactory hydrogen production efficiency. In addition, the photocatalytic efficiency also depends on the fate of photogenerated hole-electron pairs. To improve the quantum efficiency (QE) of a photocatalytic process, two sequential steps, (1) the efficient separation and transfer of the photogenerated charge carriers to the target surface reaction sites within their life time to avoid their recombination in the form of heat dissipation, and (2) the rapid implementation of reduction and oxidation reactions by those excited charges, should be promoted.

Among the various approaches, nanostructure design with welltailored band alignment provides a powerful means to the improvement of the photocatalytic activity. In the process of our study, we have also paid special attention on the nanoscale control of the material morphology and construction of functionalized nanostructures to promote charge separation and prolong the lifetime of the photoexcited charge carriers. Our discussion is principally organized around this topic, which constructs the main theme of this review.

\section{Strategies for the development of various functionalized nanostructures Nanosized functionalized morphology}

As mentioned above, absorption of photons with energy equal or higher than the band gap of the semiconductor could lead to photogenerated electrons $\left(\mathrm{e}^{-}\right)$and holes $\left(\mathrm{h}^{+}\right)$within conduction and valence bands, respectively. Generally, the photocatalytic reaction often occurs at the semiconductor surface. So the ability to accelerate the migration of photogenerated charges from the interior of the semiconductor to its surface avoiding their bulk recombination enables substantial improvement of photocatalytic efficiency. In this regard, semiconductors with nanosized functionalized morphology could be a beneficial choice. Since the first reports on mesoporous silica MCM-41 [9], inorganic oxides with controlled porosity have been extensively investigated. It is known that mesoporous photocatalysts usually exhibit high specific surface areas that can provide more surface reactive sites. The nanoscale channel walls of mesoporous photocatalysts can also facilitate the migration of photogenerated charges from bulk to surface [10]. It has been reported that porous semiconductor photocatalysts, such as $\mathrm{TiO}_{2}$ [11], $\mathrm{Nb}_{2} \mathrm{O}_{5}$ [12] and $\mathrm{Ta}_{2} \mathrm{O}_{5}$ [10], displayed much better photocatalytic properties than their bulk counterparts. Meanwhile, when semiconductor nanocrystals are dispersed in the mesoporous substrate, greatly enhanced photocatalytic properties can also be achieved. For example, dispersing $\mathrm{TiO}_{2}$ nanoclusters in MCM-41 [13] and MCM-48 [14] resulted in much higher photocatalytic activities than that of bulk $\mathrm{TiO}_{2}$ under UV light irradiation. Our work was carried out on $\mathrm{Ni}$ modified mesoporous $\mathrm{TiO}_{2}$ photocatalysts [15]. It was found 
that $\mathrm{Ni}$ ions were highly dispersed in the framework of mesoporous $\mathrm{TiO}_{2}$ resulting in enhanced hydrogen production in methanol aqueous solution compared to Ni-doped particulate $\mathrm{TiO}_{2}$. The photocatalytic activity was found to have a close relationship with the doping amount of $\mathrm{Ni}$ ions and the highest activity was obtained when the amount of $\mathrm{Ni}$ doping was $1 \%$. Here, the enhanced photocatalytic activity was attributed to doped $\mathrm{Ni}^{2+}$ ions which served as shallow trapping sites, preferentially trapping photoexcited holes. The assumption of the role of $\mathrm{Ni}^{2+}$ as shallow trapping sites could be rationalized by considering that the energy level of $\mathrm{Ni}^{2+}$ is located very close to the valence band edge of $\mathrm{TiO}_{2}$ [16]. After trapping a hole, $\mathrm{Ni}^{2+}$ will be oxidized to $\mathrm{Ni}^{3+}$. However, due to the instability of $\mathrm{Ni}^{3+}$, it will quickly return to $\mathrm{Ni}^{2+}$ again. The shallow trapping can therefore separate the arrival of photogenerated charges at the surface, so the recombination at the channel surface of mesopores could be greatly reduced. However, when the doping concentration is higher than the optimal level, or for too large particles, the exited hole can be trapped more than once, and recombine with the electron excited by another photon. An optimal dopant concentration is therefore crucial. In another example, we also synthesized Fe-doped mesoporous $\mathrm{Ta}_{2} \mathrm{O}_{5}$ that showed an enhanced activity compared to the bulk counterpart [17].

It should be pointed out that $\mathrm{TiO}_{2}$ can only respond to UV light, even metal ion doping can hardly enhance its visible light activity. Sensitization with dyes or nanocrystals is one possible approach to extend the light absorption of $\mathrm{TiO}_{2}$. Sreethawong et al. found that eosin Y-sensitized mesoporous-assembled $\mathrm{Pt} / \mathrm{TiO}_{2}$ nanocrystal photocatalysts exhibited enhanced photocatalytic hydrogen production under visible light irradiation [18]. Lee et al. also reported that CdS and CdSe nanocrystals dispersed on the internal surface of mesoporous $\mathrm{TiO}_{2}$ films could lead to the promoted photocatalytic hydrogen production under visible light [19]. We have also investigated the visiblelight-driven photocatalytic performance over a nanosized $\mathrm{WS}_{2}-$ sensitized mesoporous $\mathrm{TiO}_{2}$ photocatalyst [20]. Compared to bulk $\mathrm{TiO}_{2}$ without mesopores, more $\mathrm{WS}_{2}$ can be loaded in the mesoporous $\mathrm{TiO}_{2}$. Moreover, the mesoporous channels can prevent the light-induced detachment of $\mathrm{WS}_{2}$ nanoparticles from the substrate during the photocatalytic reaction. These factors resulted in much higher activity and better stability, as schematically illustrated in Figure 2. Here the elevation of the conduction band of nanosized $\mathrm{WS}_{2}$ due to quantum confinement effect is considered to be crucial. As the recombination of photogenerated charges within $\mathrm{TiO}_{2}$ could be neglected, the rate-determining step for photocatalytic reaction is the electron transfer from $\mathrm{TiO}_{2}$ to $\mathrm{H}^{+}$in the solution. Therefore, deposition of Pt as cocatalyst is indispensable for an efficient hydrogen evolution.

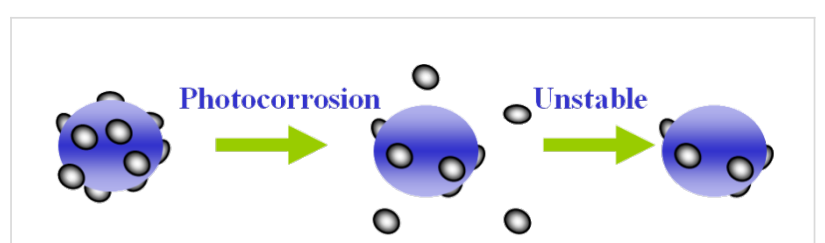

$\mathrm{WS}_{2}$ sensitized particle $\mathrm{TiO}_{2}$

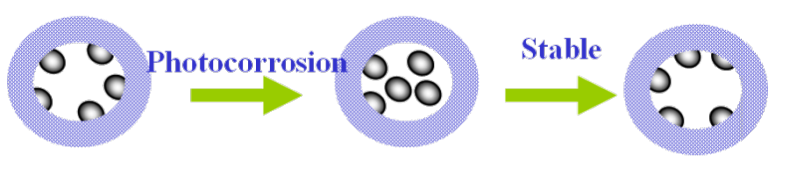

$\mathrm{WS}_{2}$ sensitized mesoporous $\mathrm{TiO}_{2}$

Figure 2: Illustration of mesoporous wall on the anti-photocorrosion of sulfide photocatalyst.

Generally, MCM-41 is not photo-reactive. But it can be activated by coupling with a semiconductor or doping a transitional metal. Figure 3 shows the proposed charge separation mechanism within a representative transitional metal-containing molecular sieve photocatalyst [21]. In principle, after excitation by visible light, photogenerated electrons are transferred from CdS to the Ti-MCM-41 substrate to conduct a reduction reaction. The holes, on the contrary, remain at the CdS to let oxidation reactions occur. We also investigated the effect taken by transitional metal doping such as $\mathrm{Cr}$ or/and $\mathrm{Ti}$ incorporation in MCM-41. Active visible light absorption sites could be generated in MCM-41 due to transitional metal doping. It was found that the highly dispersed $\mathrm{Cr}$ ions within MCM 41 could be easily excited by visible light irradiation due to the electron transfer from $\mathrm{O}^{2-}$ to $\mathrm{Cr}^{6+}$. Such excited states are active for charge transfer and thus showed a relatively high photocatalytic activity. Accordingly, the unique arrangement of the localized charges in the ordered mesoporous structure leads to a significantly prolonged life time of electron-hole pairs compared to traditional metal ion doped semiconductors.

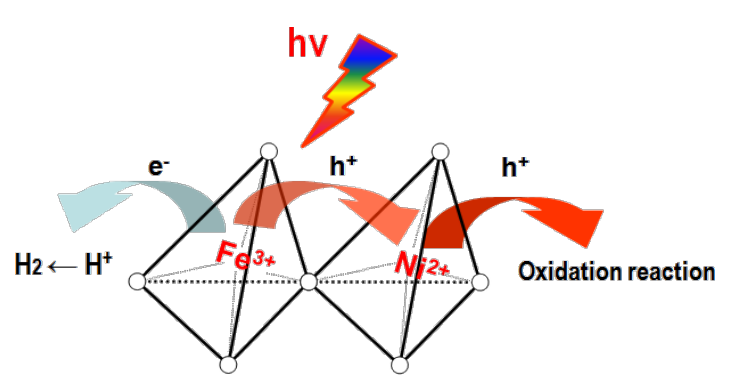

Fe/Ni-MCM-41: $\left[\mathrm{Fe}^{3+-}-\mathrm{O}-\mathrm{Ni}^{2+}\right] \stackrel{h v}{\rightarrow}\left[\mathrm{Fe}^{2+}-\mathrm{O}-\mathrm{Ni}^{3+}\right]^{*}$

Figure 3: Proposed mechanism for charge transfer in Fe/Ni co-doped MCM-41. Reprinted with permission from [21]. Copyright (2014) Elsevier. 
As was found, Cr-Ti-MCM-41 showed much higher activity than Cr-MCM-41 with similar amount of $\mathrm{Cr}$ doping for two kinds of MCM-41 material. Due to the presence of Ti, the photocatalytic mechanism of Cr-Ti-MCM-41 is quite different from that of Cr-MCM-41. For the former material, the $\mathrm{Cr}-\mathrm{O}-\mathrm{Ti}$ interaction should be responsible [22]. In this case, a second metal element introduced as a donor can closely link to the first metal element by forming an oxo bridge, which is supposed to be robust chromophore induced by metal-to-metal chargetransfer (MMCT).

\section{Nanosized functionalized surface}

A noble-metal cocatalyst, such as $\mathrm{Pt}$, is usually indispensable for many photocatalysts to achieve high photocatalytic activities for hydrogen production. It was recently reported that the valance state of platinum plays an important role in the hydrogen production efficiency, and oxidized platinum was demonstrated to be more efficient than metallic platinum as cocatalyst for hydrogen production [23,24]. Taking into account the cost of the designed photocatalyst for commercial purposes, the development of noble-metal free cocatalysts is still valued. Alternative cocatalysts such as $\mathrm{MoS}_{2}$ have been reported to be effective to enhance photocatalytic $\mathrm{H}_{2}$ evolution on CdS [25]. Low-cost WC was also used as efficient cocatalyst on CdS because of its low overpotential for hydrogen production and proper physicochemical properties [26]. The $\mathrm{Xu}$ group has studied the effect of NiS working as a cocatalyst. A simple hydrothermal method was used to synthesize NiS/CdS photocatalysts, which have a remarkably high QE of 51.3\% at $420 \mathrm{~nm}$ in lactic acid sacrificial solution [27]. Co-loading of both reduction and oxidation cocatalysts on the semiconductor was also suggested to be able to enhance the photocatalytic hydrogen production [28].

Here, it is assumed that the photocatalytic activity could be significantly improved if the cocatalysts were loaded especially on a nanosized functionalized surface. We reported the design and preparation of a highly efficient $\mathrm{Cd}_{0.5} \mathrm{Zn}_{0.5} \mathrm{~S}$ photocatalyst decorated with nanosized NiS surface heterojunctions. The hydrogen evolution rate over this photocatalyst could reach $1.4 \mathrm{mmol} / \mathrm{h}$, with a QE of $33.9 \%$. This efficiency is much higher than that of many photocatalysts containing noble metals. As shown in Figure 4, in the hybrid photocatalyst, the NiS nanoparticles can serve as electron trapping sites and extract photogenerated electrons from $\mathrm{Cd}_{0.5} \mathrm{Zn}_{0.5} \mathrm{~S}$ substrate, which finally leads to spatially separated photoreduction and oxidation reactions. NiS plays a similar role as a noble metal, which can provide active sites for proton reduction, and thus efficiently enhance the overall photocatalytic activity. Our work demonstrates that efficient and low cost photocatalytic hydrogen production can be achieved through the substitution of noble metal cocatalyst with a properly engineered surface heterojunction [29].

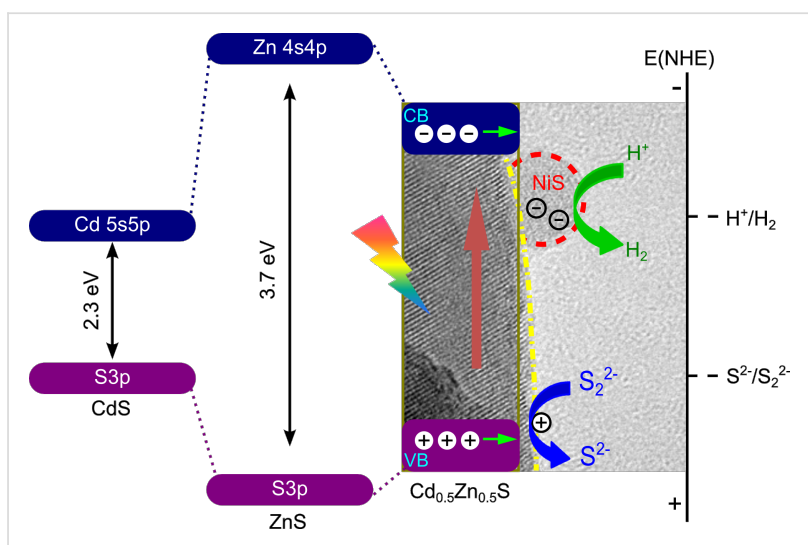

Figure 4: Proposed photocatalytic charge separation process over the band-structure controlled $\mathrm{NiS} / \mathrm{Cd}_{0.5} \mathrm{Zn}_{0.5} \mathrm{~S}$ photocatalyst. Reprinted from with permission from [29]. Copyright (2010) Elsevier.

In addition to the screening for a noble-metal free cocatalyst, we have also tried to modify the surface of the photocatalyst, aiming to form functionalized surface nanostructures. A highly active and stable CdS photocatalyst was obtained by a two-step thermal sulfuration method. Nanostep structures formed at the surface of the CdS photocatalyst, leading to a significantly improved photocatalytic activity compared to CdS prepared by traditional approaches [30]. The enrichment of $\mathrm{Pt}$ around nanostep regions revealed the preferred migration of photoexcited electrons there and the reduction $\mathrm{H}_{2} \mathrm{PtCl}_{6}$ precursor to metallic Pt. Similar nanosteps were also reported by Kudo for another metal sulfide photocatalyst [31]. Although the mechanism for the formation of nanostep structures could be different, the important role of the nanostep surface for the enhancement of photocatalytic performance was also shown.

The surface architecture of composite photocatalysts can significantly affect the photocatalytic process. For example, Jang and co-workers reported that bulky CdS decorated with $\mathrm{TiO}_{2}$ nanoparticles was much more active than bulky $\mathrm{TiO}_{2}$ decorated with CdS nanoparticles [32]. We have prepared CdS/titanate nanotubes (CdS/TNTs) photocatalysts with a unique morphology by a simple one-step hydrothermal method [33]. As schematically illustrated in Figure 5, the CdS nanoparticle was intimately enwrapped by the TNTs, resulting in a remarkably enhanced charge separation efficiency and thereby photocatalytic hydrogen production activity. The similar enwrapped structure can also be achieved for $\mathrm{Cd}_{0.5} \mathrm{Zn}_{0.5} \mathrm{~S} / \mathrm{TNTs}$ nanocomposites [34]. The QE at $420 \mathrm{~nm}$ over the nanocomposites reached $38.1 \%$ without loading any cocatalyst. Meanwhile, except for the greatly reduced toxicity by using $\mathrm{Zn}^{2+}$ instead of $\mathrm{Cd}^{2+}$ (Cd content, $\left.4.0 \mathrm{wt} \%\right), \mathrm{Cd}_{0.5} \mathrm{Zn}_{0.5} \mathrm{~S} / \mathrm{TNTs}$ also showed a 
good stability for hydrogen production. These factors are significantly beneficial for their further application in the field of solar energy conversion.

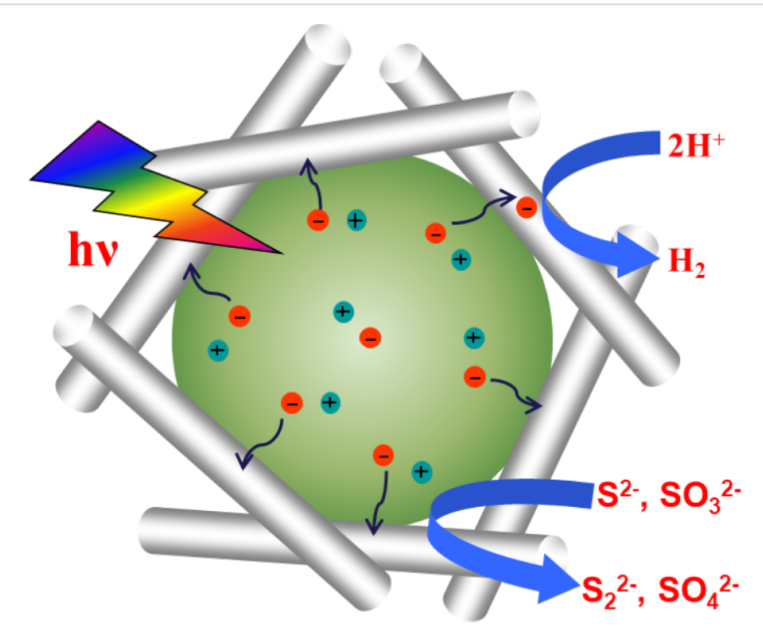

Figure 5: CdS nanoparticle enwrapped by the surrounding TNTs showed a significant enhancement of charge separation and therefore a high activity. Reproduced with permission from [33]. Copyright (2011) The Royal Society of Chemistry.

\section{Crystal facets engineering}

As we know, the surface of a given semiconductor nanocrystal usually consists of certain crystal facets that occupy specific coordination numbers and atom ratios, leading to a deviation of their band structure from their bulk counterpart. Consequently, some crystal facets have shown higher activity than other facets for many photocatalyst during a certain photocatalytic reaction [35-37]. Jang et al. found that $\mathrm{ZnO}$ nanoplates with a preferential exposure of $\mathrm{Zn}(0001)$ faces showed high photocatalytic activity [38]. Wang et al. constructed $\mathrm{ZnO}-\mathrm{CdS}$ heterostructures by employing $\mathrm{ZnO}$ rods enclosed with well-developed $\{1100\}$ and $\{0001\}$ facets to promote photocatalytic hydrogen evolution [39]. Pan et al. found that $\{001\},\{101\}$ and $\{010\}$ facets of anatase $\mathrm{TiO}_{2}$ had different photocatalytic activities. However, when partially terminated with fluorine, the three facets had similar photocatalytic activity in $\mathrm{H}_{2}$ evolution [40] Engineering the morphology of semiconductors to preferably expose active facets is therefore a promising approach, yet a big challenge to date. Selecting $\mathrm{Cu}_{2} \mathrm{WS}_{4}$ as model photocatalyst, we obtained interesting decahedral morphologies by a one-step hydrothermal method. The hydrothermal method avoids the using of toxic $\mathrm{H}_{2} \mathrm{~S}$ gas and simplifies the catalyst preparation process. Owing to the oriented growth and the presence of a large percentage of active (001) crystal planes, the photocatalyst showed high visible-light activity. The highest activity was obtained over the $\mathrm{Cu}_{2} \mathrm{WS}_{4}$ decahedrons that were hydrothermally prepared at $200{ }^{\circ} \mathrm{C}$ for $72 \mathrm{~h}$, with an QE at $425 \mathrm{~nm}$ for photocatalytic hydrogen production of $11 \%$ [41].
Regarding the stability, oxide photocatalysts would be better than sulfide and nitride photocatalysts [42]. Regrettably, oxide semiconductors frequently possess either low conduction band positions or wide band gaps, which seriously impair their suitability as photocatalysts for hydrogen production [43]. Among the very few visible-light-responsive photocatalysts, cuprous oxide $\left(\mathrm{Cu}_{2} \mathrm{O}\right)$ deserves our special attention. $\mathrm{Cu}_{2} \mathrm{O}$ is a p-type oxide semiconductor with a direct band gap of $2.0 \mathrm{eV}$. Theoretically, its light-to-hydrogen conversion efficiency can reach $18 \%$ [44]. We have thus devoted to the fabrication of $\mathrm{Cu}_{2} \mathrm{O}$ nanocrystals with controlled shape to improve its photocatalytic activity for hydrogen production. As shown in Figure 6, multifaceted $\mathrm{Cu}_{2} \mathrm{O}$ with controlled crystal facets exposure has been prepared through a facile one-step method. It was revealed that photogenerated electrons preferred to accumulate on high-indexed facets, while photogenerated holes tended to migrate to $\{100\}$ facets, leading to an efficient spatial charge separation and thereby enhanced photocatalytic hydrogen production from reforming of glucose over the $\mathrm{Cu}_{2} \mathrm{O}$ polyhedron [45]. The origin of the charge separation on different crystal facets of $\mathrm{TiO}_{2}$ has been already theoretically calculated [40]. A slight band offset was observed in both valance and conduction band between two different facets, driving the charge transfer from one facet to another. Our research indicated that a controlled fabrication of different crystal facets with separated functions, such as separated oxidation and reduction sites is one of the effective approaches to the enhancement of the activity of the existing semiconductors.
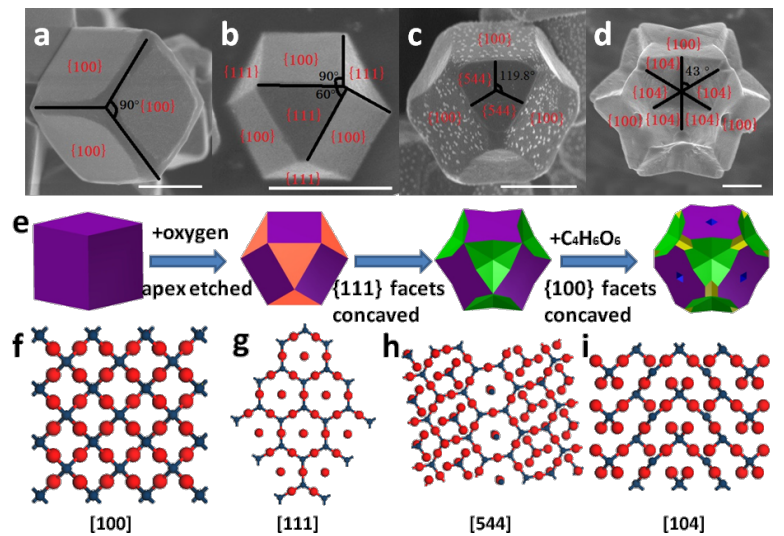

Figure 6: Morphological evolution of $\mathrm{Cu}_{2} \mathrm{O}$ prepared with different reaction times, (a) $30 \mathrm{~min}$, (b) $60 \mathrm{~min}$, (c) $90 \mathrm{~min}$ and (d) $120 \mathrm{~min}$, all scale bars are $1 \mu \mathrm{m}$. Reprinted with permission from [45]. Copyright (2014) The Royal Society of Chemistry.

Perovskite $\mathrm{ABO}_{3}$ is another group of metal oxide materials deserving in-depth exploring. Most of metal elements can be stably located in $\mathrm{ABO}_{3}$ structures, and functionalized multicomponent $\mathrm{ABO}_{3}$ materials can thus be prepared by partial substitu- 
tion of cations in A and B sites [46-48]. In this approach, we have successfully synthesized hexagonal single-crystal nanosheet-based $\mathrm{NaSbO}_{3}$ and $\mathrm{AgSbO}_{3}$ hierarchical cuboidal microclusters with exposed $\{001\}$ facets via a facile and surfactant-free hydrothermal reaction. The light absorption, charge separation and surface reaction were simultaneously optimized through the unique structure assembled from nanosheets, leading to the greatly enhanced photocatalytic activity [49]. Micro- or nanoscale surface-structuring can increase the degree of horizontal light distribution via light scattering. Otherwise, the trapped photons would be lost by direct reflection from a flat surface [50]. As shown in Figure 7, enhanced light absorption arising from multiple light reflections in the nanosheetbased hierarchical structure could be achieved over $\mathrm{NaSbO}_{3}$ and $\mathrm{AgSbO}_{3}$ photocatalysts. Meanwhile, the single-crystal nature can reduce the crystal defects leading to more efficient charge separation. The larger surface area provides more active sites for photocatalytic reaction. The exposed $\{001\}$ facets as the reactive surfaces can accelerate the redox reactions. Therefore, nanosheet-based $\mathrm{AgSbO}_{3}$ photocatalysts showed a 1.8 times higher initial $\mathrm{O}_{2}$ evolution rate than $\mathrm{AgSbO}_{3}$ photocatalysts without the hierarchical structure under visible-light irradiation.

Besides facet control, microstructure refinements of the crystal facets, such as distortion of the lattice and configuration of certain crystal facets, can also promote the charge separation and facilitate charge transfer. For example, we have investigated the effects of synthesis conditions on the structure and the photocatalytic property of $\mathrm{ZnIn}_{2} \mathrm{~S}_{4}$ [51-53]. It was discovered that the distance of $d(001) \mathrm{ZnIn}_{2} \mathrm{~S}_{4}$ could be adjusted by controlling preparation condition. The enlarged $d(001)$ spacing led to distorted $\left[\mathrm{ZnS}_{4}\right]$ and $\left[\mathrm{InS}_{4}\right]$ tetrahedrons within the $\mathrm{ZnIn}_{2} \mathrm{~S}_{4}$ crystal lattice, which in turn, generated an electrostatic field induced by a change of the dipole moments of the lattice. Such a built-in electrostatic field is obviously favorable for an efficient charge separation and hence leads to enhanced photocatalytic activity.

\section{Combined control of band structure and morphology}

In principle, a semiconductor photocatalyst should meet at least two requirements to achieve high visible-light photocatalytic activity. A high conduction band is necessary to ensure enough potential for proton reduction. It should also have a narrow band gap in order to utilize incident light to the largest extent. $\mathrm{Cd}_{1-x} \mathrm{Zn}_{x} \mathrm{~S}$, as the solid solution of $\mathrm{CdS}$ and $\mathrm{ZnS}$, has received lots of attention in recent years, owing to the tunable band structure and excellent photocatalytic properties for hydrogen production under visible light without needing noble metal cocatalysts [54]. However, the band gap of $\mathrm{Cd}_{1-x} \mathrm{Zn}_{x} \mathrm{~S}$ is not narrow enough for an efficient utilization of visible light. As reported by Kudo et al, a donor level above the valence band of $\mathrm{ZnS}$ could be formed when it is doped with $\mathrm{Ni}^{2+}$, which is responsible for the visible light response of Ni-doped $\mathrm{ZnS}$ [55]. It inspired us to further tune the band structure of $\mathrm{Cd}_{1-x} \mathrm{Zn}_{x} \mathrm{~S}$ solid solution by $\mathrm{Ni}$ doping. $\mathrm{Ni}^{2+}$-doped $\mathrm{Cd}_{1-x} \mathrm{Zn}_{x} \mathrm{~S}$ microspheres were prepared in our work. Here, the doped $\mathrm{Ni}^{2+}$ is expected to form a donor level above the valence band of $\mathrm{Cd}_{1-x} \mathrm{Zn}_{x} \mathrm{~S}$ and increase its visible light absorption. At the same time, its high conduction band can be still maintained. The enhanced photocatalytic activity was thus achieved [56]. Moreover, $\mathrm{Cd}_{1-x} \mathrm{Zn}_{x} \mathrm{~S}$ could be also modified by doping with alkali metals. We have also successfully synthesized $\mathrm{Sr}$ and $\mathrm{Ba}$ doped $\mathrm{Cd}_{1-x} \mathrm{Zn}_{x} \mathrm{~S}$ solid solution photocatalysts with improved activity [57,58]. The underlying role of the doping ions might be quite different. However, the band structure of the solid solution was similarly affected.

Mesoporous zirconium-titanium mixed phosphates (ZTP) is also a photocatalyst of interest as they not only show both cation- and anion-exchange capacity, but also can split pure

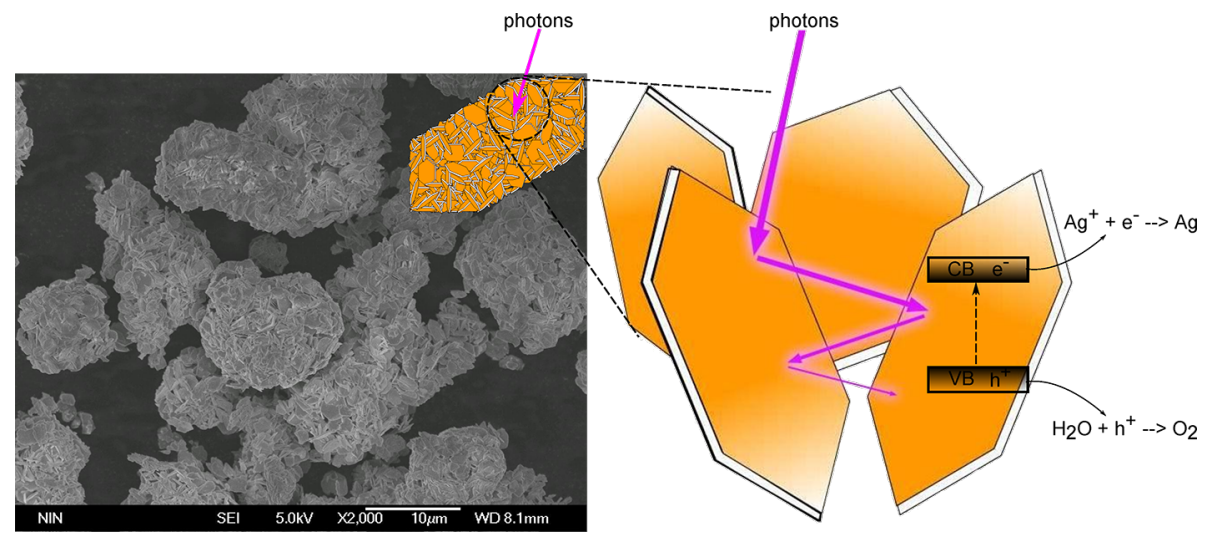

Figure 7: Enhanced light absorption ascribed to multiple light reflections in the nanosheet-based hierarchical structure. 
water for hydrogen production under UV light irradiation [59]. These properties make ZTP an ideal candidate after coupling with CdS to form visible-light composite photocatalysts. In addition, due to the adjustable $\mathrm{Zr}$ to Ti ratio, the optical properties of this material can be readily tuned. Employing ZTP as substrate and functional constituent, a novel CdS/mesoporous ZTP composite photocatalyst was successfully synthesized via the two-step thermal sulfuration method. We have found that the prepared composite photocatalyst displayed superior activity compared to that prepared by direct sulfuration at room or high temperatures. As shown in Figure 8, the conduction band of ZTP could be continuously controlled by regulating the $\mathrm{Zr} / \mathrm{Ti}$ ratio. At the optimal $\mathrm{Ti}$ to $\mathrm{Zr}$ ratio of 3, the energy difference between conduction bands of CdS and ZTP could ensure a large driving force for fluent electron transfer from CdS to ZTP, while the electron localized on the ZTP substrate is still sufficiently active for hydrogen evolution. Combination of CdS with band adjustable mesoporous ZTP could thus inherit the advantage of ZTP in terms of both morphology and band structure. The QE of this composite photocatalyst at $420 \mathrm{~nm}$ was determined to be $27.2 \%$ [60].

In addition, we also prepared flower like Ni-doped $\mathrm{ZnIn}_{2} \mathrm{~S}_{4}$ with plenty of curved nanosheets using hydrothermal method, as shown in Figure 9. It was found that there existed an optimal $\mathrm{Ni}$ doping concentration, i.e., $0.3 \mathrm{wt} \%$, for the highest hydro- gen production efficiency. Only Ni ions occupying crystal lattice sites of $\mathrm{ZnIn}_{2} \mathrm{~S}_{4}$ were found to be active. A higher $\mathrm{Ni}$ doping is not effective due to the limited solubility of $\mathrm{Ni}$ in the lattice of $\mathrm{ZnIn}_{2} \mathrm{~S}_{4}$ and could be detrimental owing to the coverage of surface reaction sites. The existence of thin nanoscale sheets is regarded to be important for its increased activity. $\mathrm{Ni}^{2+}$ ions were also expected to be shallow trapping sites, which could enhance the charge separation [61]. Similar combined control of band structure and morphology were also carried out by other groups. Kudo and co-workers prepared layered $\mathrm{AGa}_{2} \mathrm{In}_{3} \mathrm{~S}_{8}(\mathrm{~A}=\mathrm{Cu}$ or $\mathrm{Ag})$ by a solid state method for photocatalytic hydrogen production. Both $\mathrm{CuGa}_{2} \mathrm{In}_{3} \mathrm{~S}_{8}$ $(1.91 \mathrm{eV})$ and $\mathrm{AgGa}_{2} \mathrm{In}_{3} \mathrm{~S}_{8}(2.27 \mathrm{eV})$ showed a quite high photocatalytic activity [62]. Chen and co-workers synthesized hierarchical $\mathrm{ZnS}-\mathrm{In}_{2} \mathrm{~S}_{3}-\mathrm{CuS}$ nanospheres with a nanoporous structure. A high QE of $22.6 \%$ at $420 \mathrm{~nm}$ is achieved without loading cocatalysts due to their high crystallinity, high surface area and unique microstructure [63].

As the crystal size of the semiconductor is close to the exciton Bohr radius, its bandgap can be enlarged with a reduced crystal size due to the quantum confinement effect. Therefore, we have synthesized $\mathrm{Co}_{3} \mathrm{O}_{4}$ quantum dots $(3-4 \mathrm{~nm})$ via a facile reverse micelle method for the first time. $\mathrm{Co}_{3} \mathrm{O}_{4}$ has been widely employed as a photocatalyst for oxygen production due to its nontoxicity, low cost and narrow band gap $(2.1 \mathrm{eV})$. However,

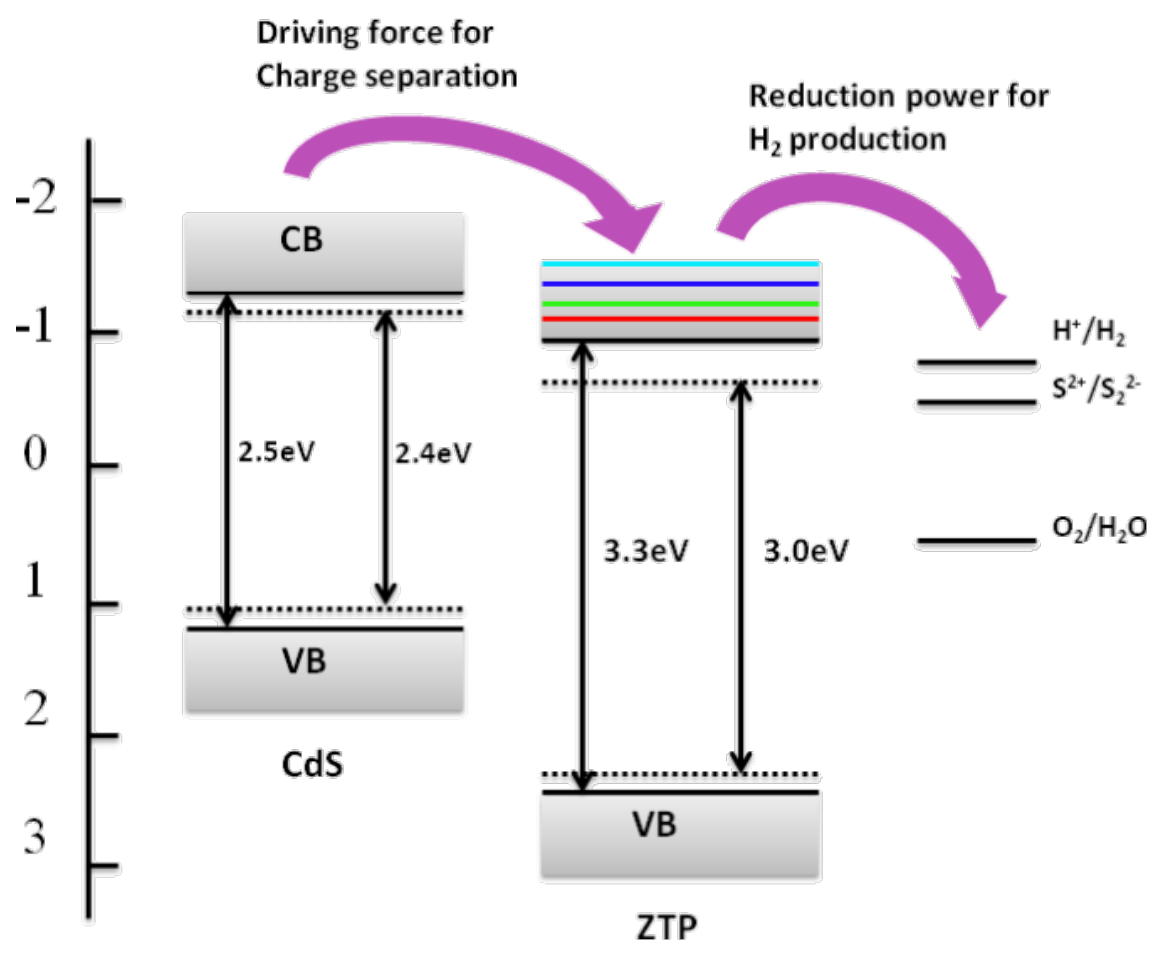

Figure 8: Schematic illustration for the charge separation in CdS/mesoporous ZTP. Reprinted with permission from [60]. Copyright (2007) The American Chemical Society. 


\section{$\mathrm{E}(\mathrm{NHE})$}
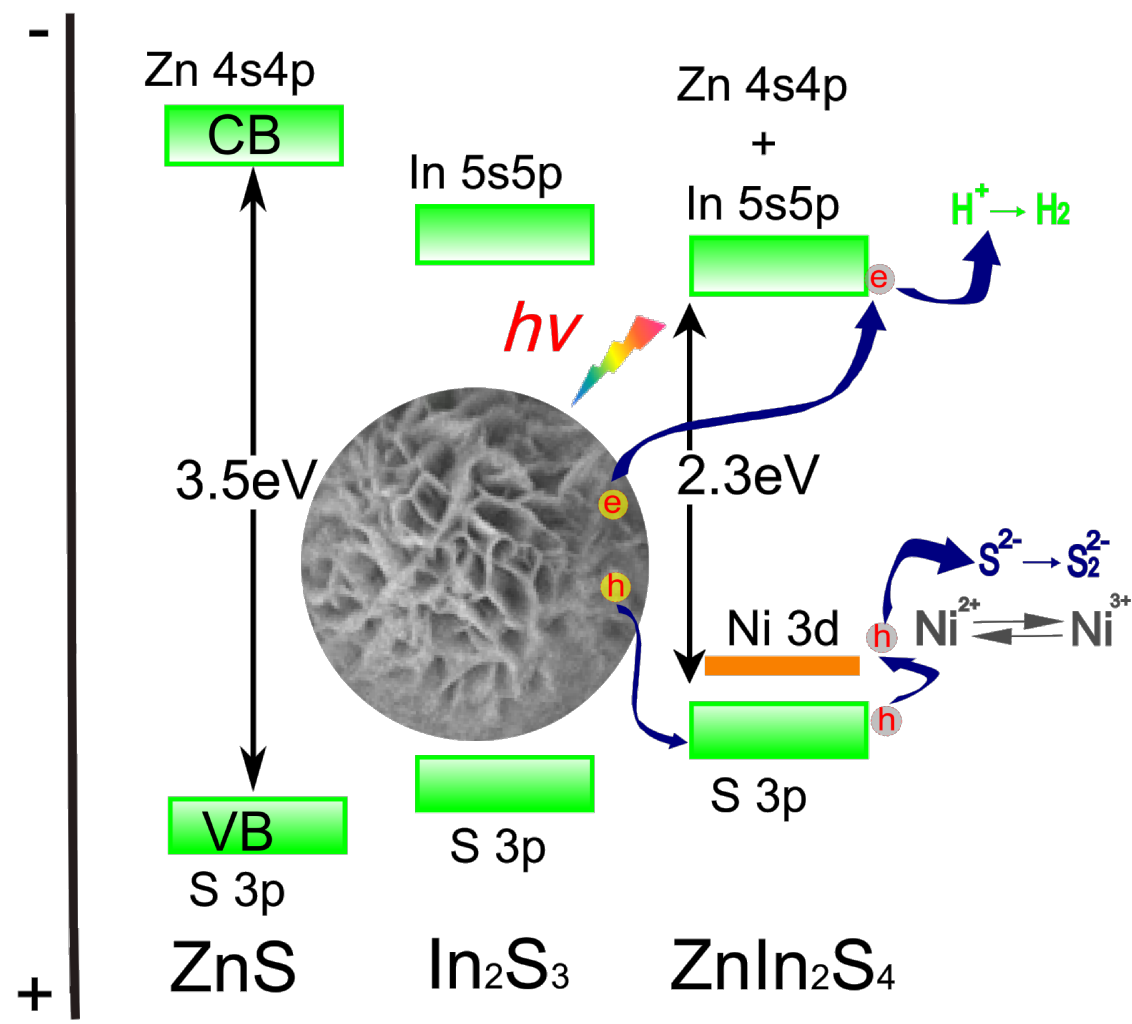

Figure 9: Photocatalytic mechanism over Ni-doped $\mathrm{ZnIn}_{2} \mathrm{~S}_{4}$ with plenty of curved nanosheets. Reprinted with permission from [61]. Copyright (2010), Springer.

photocatalytic hydrogen production could not be achieved, because the conduction band edge of bulk $\mathrm{Co}_{3} \mathrm{O}_{4}$ is not negative enough to reduce $\mathrm{H}^{+}$. Compared with bulk $\mathrm{Co}_{3} \mathrm{O}_{4}, \mathrm{Co}_{3} \mathrm{O}_{4}$ quantum dots have a wider bandgap. Valence-band XPS spectra showed that the valance band maxima (VBM) of $\mathrm{Co}_{3} \mathrm{O}_{4}$ quantum dots and bulk $\mathrm{Co}_{3} \mathrm{O}_{4}$ are almost at the same position, implying that the enlarged bandgap of $\mathrm{Co}_{3} \mathrm{O}_{4}$ quantum dots was mainly ascribed to the conduction band minimum (CBM) upshift. Due to the negative shift of the conduction band, $\mathrm{Co}_{3} \mathrm{O}_{4}$ quantum dots can split pure water into $\mathrm{O}_{2}$ and $\mathrm{H}_{2}$ stoichiometrically under visible light irradiation without any cocatalyst. This is the first report to date of $\mathrm{Co}_{3} \mathrm{O}_{4}$ photocatalysts capable of splitting pure water, which provides a new route to the development of nanosized photocatalysts for water splitting [64].

\section{From single heterojunction to long range ordered homojunction}

Semiconductor heterostructures can be engineered to combine functionalities that result from the bulk properties of their constituent phases with properties that are directly related to the electronic and atomic character of their interfaces [65]. The concept has been proven in photovoltaic cells and optoelec- tronic devices, where junction-type semiconductors show greatly improved efficiency compared with junction-free semiconductors [66]. Depending on the band gaps and the electronic affinity of semiconductors, semiconductor heterostructures can be divided into three different cases: type-I, type-II and type-III band alignment [67]. In a type-II band alignment, the band offsets in the conduction and valance bands go in the same direction, resulting in a band bending at the interface between two semiconductors. Such band bending consequently induces a built-in field, which drives the vectorial migration of the photogenerated charges at the interface, leading to a spatial separation of $\mathrm{e}^{-}$and $\mathrm{h}^{+}$on different sides of the heterojunction. Most type-II heterojunctions have been obtained from two different semiconductors $[68,69]$. Matching of two semiconductor materials with both their band positions and crystal lattices is the key challenge of this strategy. Most recently, successful efforts have been made to fabricate heterojunctions of different phases of the same material. Bao and co-workers prepared CdS photocatalysts with different phases for photocatalytic hydrogen production [70]. Interestingly, a higher photocatalytic activity is observed from the composite of hexagonal and cubic $\mathrm{CdS}$ as compared to single hexagonal or cubic 
CdS. However, the relation of electronic interaction between different phases with the photocatalytic activities was not discussed in their study [70]. Li and co-workers have demonstrated the greatly enhanced photocatalytic overall water splitting over an $\alpha-\beta$ phase junction of $\mathrm{Ga}_{2} \mathrm{O}_{3}$ [71]. The improved photocatalytic activity results from the efficient charge separation and transfer across the $\alpha-\beta$ phase junctions of the $\mathrm{Ga}_{2} \mathrm{O}_{3}$ particles.

We have found that spherical twin-containing noble-metal-free $\mathrm{Cd}_{0.5} \mathrm{Zn}_{0.5} \mathrm{~S}$ is a superb photocatalyst for hydrogen production, showing a QE of $43 \%$ at $425 \mathrm{~nm}$ [72]. However, the low density of twin planes and the insufficient control of the crystal shape in these catalysts inspired us to further improve their photocatalytic efficiency by fabricating more effective junctions. Recently, we reported a twinned $\mathrm{Cd}_{0.5} \mathrm{Zn}_{0.5} \mathrm{~S}$ anisotropic nanocrystal with controllable aspect ratios and a high percentage of long-range ordered twin planes. As shown in Figure 10, the densely distributed rotational twin planes are found to be parallel to each other and perpendicular to the $<111>$ direction. More interestingly, zinc-blende (ZB) and wurtzite (WZ) segments alternatively occur along the $<111>$ direction of the designed material, resulting in its unique optical and electrical properties. It was also shown that type-II staggered band alignment between $\mathrm{WZ}$ and ZB segments resulted in an immense number of homojunctions in a specific dimension. Unlike the well-known heterojunctions, no foreign-atom doping or combination was required for the formation of these junctions, resulting in the homogeneity of the materials themselves and thereby enabling us to engineer the necessary semiconductor band structures

\section{Twin-induced homojunctions}

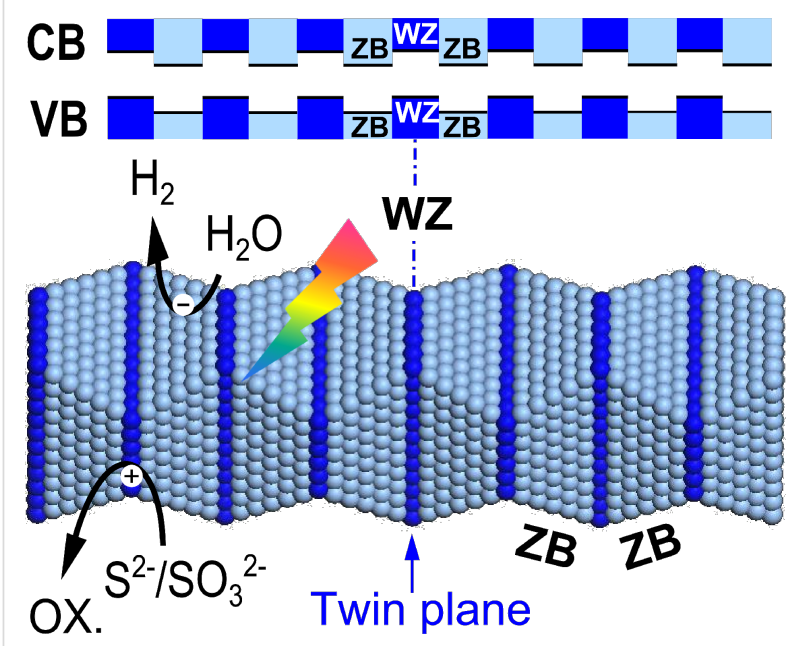

Figure 10: Illustration of twin-induced 1D long range ordered homojunctions over $\mathrm{Cd}_{0.5} \mathrm{Zn}_{0.5} \mathrm{~S}$ with alternative zinc-blende (ZB) and wurtzite (WZ) segments. more exactly. Notably, it was possible to raise the QE for the photocatalytic hydrogen formation to $62 \%$ because of the improved efficiency in charge separation [73]. These dense homojunctions are clearly superior to the single homojunction formed by introduction of a thin p-type layer on n-type $\alpha-\mathrm{Fe}_{2} \mathrm{O}_{3}$ and creating a built-in field as reported in [74]. We suggest that our results have the potential to lead to a new general method for the synthesis of new, highly active photocatalysts by the application of our method to other semiconducting materials.

\section{Conclusion}

With the rapid development of creative nanomaterials for new energy applications [75], the nanostructures have also been found essential for achieving high efficient photocatalysts and cocatalysts. The heart of the study is the nanostructure design from functionalized nanosized morphology and surfaces of relatively larger scale to the precise tuning of crystal facets and junctions in much smaller scale. In the process of our study, we firstly attempted the simple combination of two or more semiconductors both physically and chemically. Parallel to this advancement, nanosized functionalized morphology and surfaces are employed in these hetero-combinations to obtain enhanced charge generation and separation. We then elaborate on the combined control of band structure and morphology to reveal the synergistic effects by coupling two or three kinds of modifications in one semiconductor. In this case, enhanced matching of two components in one hybrid photocatalyst, in terms of morphologic contact, band structure and crystal lattices could be achieved. Especially, various heterostructure architectures with combined functionality of the constituent phases are suggested to be promising to address the primary requirements for an efficient photocatalytic generation of hydrogen by using solar energy: visible-light activity, chemical stability, appropriate band-edge characteristics, and potential for low-cost fabrication. Finally, we highlight the heterostructure design at a precise nanoscale control, such as materials of same composition but different phases and/or from heterojunction to homojunction engineering. As has been demonstrated by our twinned $\mathrm{Cd}_{0.5} \mathrm{Zn}_{0.5} \mathrm{~S}$, an ordered homojunction is much effective than a disordered one. Co-existence of two phases in same semiconducting material in an ordered way, preferably with type-II band alignment, is therefore recommended to be the most desired nanostructures enabling photo-chemistry or -electricity conversion. How to construct such nanostructures in a low-cost and time-efficient way is the challenge ahead.

\section{Acknowledgements}

The authors gratefully acknowledge the financial support from the National Natural Science Foundation of China (No. 51236007, 21276206 and 51121092) and National Basic Research Program of China (No. 2009CB220000). 


\section{References}

1. Chen, X. B.; Shen, S. H.; Guo, L. J.; Mao, S. S. Chem. Rev. 2010, 110, 6503-6570. doi:10.1021/cr1001645

2. Maeda, K.; Teramura, K.; Lu, D.; Takata, T.; Saito, N.; Inoue, Y.; Domen, K. Nature 2006, 440, 295. doi:10.1038/440295a

3. Kudo, A.; Miseki, Y. Chem. Soc. Rev. 2009, 38, 253-278. doi:10.1039/b800489g

4. Jing, D. W.; Yao, S.; Cheng, P.; Liu, M.; Shi, J. W.; Zhao, L.; Yan, W.; Guo, L. J. AIChE J. 2012, 58, 3593-3596. doi:10.1002/aic.13833

5. Jing, D. W.; Liu, H.; Zhang, X. H.; Zhao, L.; Guo, L. J. Energy Convers. Manage. 2009, 50, 2919-2926. doi:10.1016/j.enconman.2009.07.012

6. Jing, D. W.; Guo, L. J.; Zhao, L.; Zhang, X. M.; Liu, H.; Li, M. T.; Shen, S. H.; Liu, G. J.; Hu, X. W.; Zhang, X. H.; Zhang, K.; Ma, L. J.; Guo, P. H. Int. J. Hydrogen Energy 2010, 35, 7087-7097. doi:10.1016/j.ijhydene.2010.01.030

7. Fujishima, A.; Honda, K. Nature 1972, 238, 37-38. doi:10.1038/238037a0

8. Van Gerven, T.; Mul, G.; Moulijn, J.; Stankiewicz, A. Chem. Eng. Process. 2007, 46, 781-789. doi:10.1016/j.cep.2007.05.012

9. Kresge, C. T.; Leonowicz, M. E.; Roth, W. J.; Vartuli, J. C.; Beck, J. S. Nature 1992, 359, 710-712. doi:10.1038/359710a0

10. Takahara, Y.; Konde, J.; Takata, N. T.; Lu, D.; Domen, K. Chem. Mater. 2001, 13, 1194-1199. doi:10.1021/cm000572i

11. Jitputti, J.; Pavasupree, S.; Suzuki, Y.; Yoshikawa, S. J. Solid State Chem. 2007, 180, 1743-1749. doi:10.1016/j.jssc.2007.03.018

12. Chen, X.; Yu, T.; Fan, X.; Zhang, H.; Li, Z.; Ye, J.; Zou, Z. Appl. Surf. Sci. 2007, 253, 8500-8506. doi:10.1016/j.apsusc.2007.04.035

13. Liu, S.-H.; Huang, Y.-J.; Lin, K.-S.; Hsiao, M.-C. Energy Sources 2003, 25, 591-596. doi:10.1080/00908310390195651

14. Zhao, D.; Budhi, S.; Rodriguez, A.; Koodali, R. T. Int. J. Hydrogen Energy 2010, 35, 5276-5283. doi:10.1016/j.ijhydene.2010.03.087

15. Jing, D. W.; Zhang, Y. J.; Guo, L. J. Chem. Phys. Lett. 2005, 415, 74-78. doi:10.1016/j.cplett.2005.08.080

16. Nishikawa, T.; Nakajima, T.; Shinohara, Y. J. Mol. Struct. 2001, 545, 67-74. doi:10.1016/S0166-1280(01)00394-3

17. Jing, D. W.; Guo, L. J. J. Phys. Chem. Solids 2007, 68, 2363-2369. doi:10.1016/j.jpcs.2007.07.045

18. Sreethawong, T.; Junbua, C.; Chavadej, S. J. Power Sources 2009, 190, 513-524. doi:10.1016/j.jpowsour.2009.01.054

19. Lee, Y.; Chi, C.; Liau, S. Chem. Mater. 2010, 22, 922-927. doi:10.1021/cm901762h

20. Jing, D. W.; Guo, L. J. Catal. Commun. 2007, 8, 795-799. doi:10.1016/j.catcom.2006.09.009

21. Shen, S. H.; Chen, J.; Koodali, R. T.; Hu, Y. F.; Xiao, Q. F.; Zhou, J. G.; Wang, X. X.; Guo, L. J. Appl. Catal., B 2014, 150-151, 138-146. doi:10.1016/j.apcatb.2013.12.014

22. Shen, S. H.; Guo, L. J. Catal. Today 2007, 129, 414-420. doi:10.1016/j.cattod.2006.08.070

23. Li, Y. H.; Xing, J.; Chen, Z. J.; Li, Z.; Tian, F.; Zheng, L. R.; Wang, H. F.; Hu, P.; Zhao, H. J.; Yang, H. G. Nat. Commun. 2013, 4, 2500. doi:10.1038/ncomms 3500

24. Xing, J.; Jiang, H. B.; Chen, J. F.; Li, Y. H.; Wu, L.; Yang, S.; Zheng, L. R.; Wang, H. F.; Hu, P.; Zhao, H. J.; Yang, H. G. J. Mater. Chem. A 2013, 1, 15258-15264. doi:10.1039/c3ta13167j
25. Zong, X.; Yan, H.; Wu, G.; Ma, G.; Wen, F.; Wang, L.; Li, C. J. Am. Chem. Soc. 2008, 130, 7176-7177. doi:10.1021/ja8007825

26. Jang, J. S.; Ham, D. J.; Lakshminarasimhan, N.; Choi, W.; Lee, J. S. Appl. Catal., A 2008, 346, 149-154. doi:10.1016/j.apcata.2008.05.020

27. Zhang, W.; Wang, Y. B.; Wang, Z.; Zhong, Z. Y.; Xu, R. Chem. Commun. 2010, 46, 7631-7633. doi:10.1039/c0cc01562h

28. Yang, J. H.; Wang, D. E.; Han, H. X.; Li, C. Acc. Chem. Res. 2013, 46, 1900-1909. doi:10.1021/ar300227e

29. Li, N. X.; Zhou, B. Y.; Guo, P. H.; Zhou, J. C.; Jing, D. W. Int. J. Hydrogen Energy 2013, 38, 11268-11277. doi:10.1016/j.ijhydene.2013.06.067

30. Jing, D. W.; Guo, L. J. J. Phys. Chem. B 2006, 110, 11139-11145. doi:10.1021/jp060905k

31. Tsuji, I.; Kato, H.; Kobayashi, H.; Kudo, A. J. Am. Chem. Soc. 2004, 126, 13406-13413. doi:10.1021/ja048296m

32. Jang, J. S.; Li, W.; Oh, S. H.; Lee, J. S. Chem. Phys. Lett. 2006, 425 278-282. doi:10.1016/j.cplett.2006.05.031

33. Chen, Y. B.; Wang, L. Z.; Lu, G. Q.; Yao, X. D.; Guo, L. J. J. Mater. Chem. 2011, 21, 5134-5141. doi:10.1039/c0jm03945d

34. Chen, Y. B.; Guo, L. J. J. Mater. Chem. 2012, 22, 7507-7514. doi:10.1039/c2jm16797b

35. Yang, H. G.; Sun, C. H.; Qiao, S. Z.; Zou, J.; Liu, G.; Smith, S. C.; Cheng, H. M.; Lu, G. Q. Nature 2008, 453, 638-641. doi:10.1038/nature06964

36. Li, R.; Zhang, F.; Wang, D.; Yang, J.; Li, M.; Zhu, J.; Zhou, X.; Han, H.; Li, C. Nat. Commun. 2013, 4, 1432. doi:10.1038/ncomms2401

37. Martin, D. J.; Umezawa, N.; Chen, X. W.; Ye, J. H.; Tang, J. W. Energy Environ. Sci. 2013, 6, 3380-3386. doi:10.1039/c3ee42260g

38. Jang, E. S.; Won, J. H.; Hwang, S. J.; Choy, J. H. Adv. Mater. 2006, 18, 3309-3312. doi:10.1002/adma.200601455

39. Wang, X. W.; Liu, G.; Chen, Z. G.; Li, F.; Wang, L. Z.; Lu, G. Q.; Cheng, H. M. Chem. Commun. 2009, 3452. doi:10.1039/b904668b

40. Pan, J.; Liu, G.; Lu, G. Q.; Cheng, H. M. Angew. Chem., Int. Ed. 2011, 50, 2133-2137. doi:10.1002/anie.201006057

41. Jing, D. W.; Liu, M. C.; Chen, Q. Y.; Guo, L. J. Int. J. Hydrogen Energy 2010, 35, 8521-8527. doi:10.1016/j.jjhydene.2010.04.170

42. Huang, G.; Shi, R.; Zhu, Y. J. Mol. Catal. A 2011, 348, 100-105. doi:10.1016/j.molcata.2011.08.013

43. Luo, F.; Wu, D.; Gao, L.; Lian, S.; Wang, E.; Kang, Z.; Lan, Y.; Xu, L. J. Cryst. Growth 2005, 285, 534-540. doi:10.1016/j.jcrysgro.2005.09.032

44. Paracchino, A.; Laporte, V.; Sivula, K.; Grätzel, M.; Thimsen, E. Nat. Mater. 2011, 10, 456-461. doi:10.1038/nmat3017

45. Zhang, L. Z.; Shi, J. W.; Liu, M. C.; Jing, D. W.; Guo, L. J. Chem. Commun. 2014, 50, 192-194. doi:10.1039/c3cc46423g

46. Shi, J. W.; Guo, L. J. Prog. Nat. Sci. Mater. Int. 2012, 22, 592-615. doi:10.1016/j.pnsc.2012.12.002

47. Shi, J. W.; Ye, J.; Ma, L.; Ouyang, S.; Jing, D.; Guo, L. J. Chem.-Eur. J. 2012, 18, 7543-7551. doi:10.1002/chem.201102807

48. Shi, J. W.; Ye, J. H.; Zhou, Z. H.; Li, M. T.; Guo, L. J. Chem.-Eur. J. 2011, 17, 7858-7867. doi:10.1002/chem.201003755

49. Shi, J. W.; Ye, J. H.; Li, Q. Y.; Zhou, Z. H.; Tong, H.; Xi, G. C.; Guo, L. J. Chem.-Eur. J. 2012, 18, 3157-3162. doi:10.1002/chem.201102214

50. Osterloh, F. E. Chem. Soc. Rev. 2013, 42, 2294-2320. doi:10.1039/c2cs35266d

51. Shen, S. H.; Zhao, L.; Guo, L. J. J. Phys. Chem. Solids 2008, 69, 2426-2432. doi:10.1016/j.jpcs.2008.04.035

52. Shen, S. H.; Zhao, L.; Guo, L. J. Int. J. Hydrogen Energy 2008, 33, 4501-4510. doi:10.1016/j.ijhydene.2008.05.043 
53. Shen, S. H.; Zhao, L.; Guo, L. J. Mater. Res. Bull. 2009, 44, 100-105. doi:10.1016/j.materresbull.2008.03.027

54. Zhang, K.; Guo, L. J. Catal. Sci. Technol. 2013, 3, 1672-1690. doi:10.1039/c3cy00018d

55. Kudo, A.; Kato, H.; Tsuji, I. Chem. Lett. 2004, 33, 1534-1539. doi:10.1246/cl.2004.1534

56. Zhang, X. H.; Jing, D. W.; Liu, M. C.; Guo, L. J. Catal. Commun. 2008, 9, 1720-1724. doi:10.1016/j.catcom.2008.01.032

57. Zhang, K.; Jing, D. W.; Chen, Q.; Guo, L. J. Int. J. Hydrogen Energy 2010, 35, 2048-2057. doi:10.1016/j.ijhydene.2009.12.143

58. Zhang, K.; Zhou, Z. H.; Guo, L. J. Int. J. Hydrogen Energy 2011, 36 , 9469-9478. doi:10.1016/j.ijhydene.2011.05.058

59. Kapoor, M. P.; Inagaki, S.; Yoshida, H. J. Phys. Chem. B 2005, 109, 9231-9238. doi:10.1021/jp045012b

60. Jing, D. W.; Guo, L. J. J. Phys. Chem. C 2007, 111, 13437-13441. doi:10.1021/jp071700u

61. Jing, D. W.; Liu, M. C.; Guo, L. J. Catal. Lett. 2010, 140, 167-171. doi:10.1007/s10562-010-0442-9

62. Kaga, H.; Saito, K.; Kudo, A. Chem. Commun. 2010, 46, 3779-3781. doi:10.1039/b927362j

63. Li, Y. X.; Chen, G.; Wang, Q.; Wang, X.; Zhou, A. K.; Shen, Z. Y. Adv. Funct. Mater. 2010, 20, 3390-3398. doi:10.1002/adfm.201000604

64. Zhang, N.; Shi, J. W.; Mao, S. S.; Guo, L. J. Chem. Commun. 2014, 50 2002-2004. doi:10.1039/c3cc48026g

65. Kronawitter, C. X.; Vayssieres, L.; Shen, S. H.; Guo, L. J.; Wheeler, D. A.; Zhang, J. Z.; Antoun, B. R.; Mao, S. S. Energy Environ. Sci. 2011, 4, 3889-3899. doi:10.1039/c1ee02186a

66. Jang, J. S.; Kim, H. G.; Lee, J. S. Catal. Today 2012, 185, 270-277. doi:10.1016/j.cattod.2011.07.008

67. Wang, Y. J.; Wang, Q. S.; Zhan, X. Y.; Wang, F. M.; Safdar, M.; He, J. Nanoscale 2013, 5, 8326-8339. doi:10.1039/c3nr01577g

68. McDaniel, H.; Heil, P. E.; Tsai, C. L.; Kim, K. K.; Shim, M. ACS Nano 2011, 5, 7677-7683. doi:10.1021/nn2029988

69. Guo, P. H.; Jiang, J. G.; Shen, S. H.; Guo, L. J. Int. J. Hydrogen Energy 2013, 38, 13097-13103. doi:10.1016/j.ijhydene.2013.01.184

70. Bao, N.; Shen, L.; Takata, T.; Domen, K.; Gupta, A.; Yanagisawa, K.; Grimes, C. A. J. Phys. Chem. C 2007, 111, 17527-17534. doi:10.1021/jp076566s

71. Wang, X.; Xu, Q.; Li, M. R.; Shen, S.; Wang, X. L.; Wang, Y. C.; Feng, Z. C.; Shi, J. Y.; Han, H. X.; Li, C. Angew. Chem., Int. Ed. 2012, 51, 13089-13092. doi:10.1002/anie.201207554

72. Liu, M.; Wang, L.; Lu, G.; Yao, X.; Guo, L. Energy Environ. Sci. 2011, 4, 1372-1378. doi:10.1039/c0ee00604a

73. Liu, M. C.; Jing, D. W.; Zhou, Z. H.; Guo, L. J. Nat. Commun. 2013, 4, 2278. doi:10.1038/ncomms3278

74. Lin, Y. J.; Xu, Y.; Mayer, M. T.; Simpson, Z. I.; McMahon, G.; Zhou, S.; Wang, D. J. Am. Chem. Soc. 2012, 134, 5508-5511. doi:10.1021/ja300319g

75. Mao, S. S.; Chen, X. Int. J. Energy Res. 2007, 31, 619-636. doi:10.1002/er.1283

\section{License and Terms}

This is an Open Access article under the terms of the Creative Commons Attribution License

(http://creativecommons.org/licenses/by/2.0), which permits unrestricted use, distribution, and reproduction in any medium, provided the original work is properly cited.

The license is subject to the Beilstein Journal of Nanotechnology terms and conditions:

(http://www.beilstein-journals.org/bjnano)

The definitive version of this article is the electronic one which can be found at:

doi:10.3762/bjnano.5.113 